\title{
A Note on the Kelvin Effect in 100Cr6 Steel with Application to Identification of the Elastoplastic Limit
}

\author{
Håkan Hallberg \\ Division of Solid Mechanics, Lund University, P.O. Box 118, 22100 Lund, Sweden \\ Correspondence should be addressed to Håkan Hallberg, hakan.hallberg@solid.lth.se
}

Received 8 December 2011; Accepted 10 January 2012

Academic Editors: R. J. Abergel and P. J. Masset

Copyright ( 2012 Håkan Hallberg. This is an open access article distributed under the Creative Commons Attribution License, which permits unrestricted use, distribution, and reproduction in any medium, provided the original work is properly cited.

Experimental and analytical results are presented regarding the temperature evolution in 100Cr6 steel under uniaxial loading. Differently heat-treated conditions of the material are studied at different strain rates. In the annealed state, the materials exhibits a pronounced initial yield stress as it passes from the elastic region to the plastic through discontinuous yielding. In contrast, the quenched and tempered material yields continuously. The focus of the paper is on the temperature decrease during elastic deformation that precedes the more pronounced heating due to inelastic dissipation once the elastoplastic limit stress is surpassed. The applicability of the maximum temperature decrease in the elastic regime as a replacement for the commonly used $0.2 \%$ strain measure to define the elastoplastic limit is discussed. For $100 \mathrm{Cr} 6$ steel, the $0.2 \%$-strain measure is found, in some cases, to overestimate the initial yield stress by $50 \mathrm{MPa}$. The drop in temperature corresponding to the shift from elastic to inelastic material behavior is experimentally determined and compared to predictions by the Kelvin formula which in the current study give a maximum $50 \%$ error.

\section{Introduction}

The thermal conditions will continuously change during deformation of a solid material. Considering uniaxial loading of metals and steel, the deformation can with close agreement initially be considered to be linearly elastic. When the elastic limit is reached and yielding occurs, kinks and dislocations that were initially sessile will become mobile, increasingly organized, and also multiply in number. This dynamic instability in the material marks the change in deformation behavior from elastic to plastic, that is, from reversible to irreversible. The elastic limit is in many cases hard to precisely determine from material tests, motivating the common approximation of the initial yield stress by $\sigma_{0.2}$, the stress at $0.2 \%$ inelastic strain. It has been suggested in, for example $[1,2]$ that this ambiguous measure be replaced by considering the thermal behavior of the material. During elastic- and presumably reversible-deformation of a solid material, the thermoelastic interaction will lead to a decrease in temperature. It is suggested that the temperature will reach its lowest level in conjunction with the initial yielding of the material. This temperature decrease is in the order of
$1 \mathrm{~K}$ in metals and steel while polymers can exhibit a more pronounced temperature drop, cf. [3]. Once the deformation passes from the elastic to the plastic region, the internal plastic work will lead to internal heating that rapidly masks the thermoelastic cooling and reverse the temperature trend. The maximum drop in temperature thus indicates the point at which the thermoelastic cooling and the heating due to plastic dissipation is equal in magnitude.

The thermoelastic temperature decrease was addressed and quantified already by Kelvin, who related the adiabatic temperature change during deformation of a solid to the coefficient of thermal expansion, the density, and the specific heat of the material. A slightly different point of view was put forward by $[4,5]$, who recognized a constant ratio between the coefficient of thermal expansion and the specific heat of a material. Different expressions for the corresponding Grüneisen parameter have since emerged in literature. The relation between the Kelvin formulation and the Grüneisen parameter is discussed in [6] in relation to for example, material selection in strain gages. The temperature decrease during elastic deformation has also been discussed in $[1,2,7]$ in connection with the prediction of the elastoplastic limit. 
Thermoelastic effects in connection with measurements of the subsequent stored energy of cold work are discussed in relation to experiments in [8-13]. Similar studies were performed in $[14,15]$ which focussed on the temperature evolution in front of a propagating crack. Thermoelastic material behavior in shape-memory alloys during cyclic loading is discussed in [16,17] and in rock material in [18].

The present work includes both experimental and analytical results on the thermoelastic behavior of 100Cr6 steel under uniaxial loading. This steel is frequently used in engineering applications, for example, in bearing applications. Differently heat-treated conditions of the material are used and the specimens are subjected to different strain rates. The material-in the annealed condition-exhibits a distinct initial yield stress followed by discontinuous yielding, resulting in an uncertain corresponding yield strain. This yield plateau is absent in the quenched and tempered 100Cr6 that is also studied here. Considering the thermomechanical coupling, theoretical assessments are made of the temperature drop during elastic deformation of the material and its applicability to prediction of the elastoplastic limit.

A discussion is given initially regarding the calculation of the thermoelastic temperature drop after which experimental findings are discussed with a comparison of experimental data and theoretical predictions.

\section{Thermodynamic Considerations}

The first and second laws of thermodynamics allow the heat equation to be written as

$$
\rho c \dot{\theta}=\dot{W}_{p}+\dot{W}_{e},
$$

where $\dot{W}_{p}$ and $\dot{W}_{e}$ are the rates of plastic and elastic work, respectively. The mass density is denoted by $\rho$, the specific heat by $c$, and the absolute temperature by $\theta$. Noting that $\dot{W}_{p}$ is zero during elastic deformation, the thermoelastic behavior is determined from $\dot{W}_{\mathrm{e}}$. Since the thermal strains are assumed to be purely volumetric, $\dot{W}_{e}$ will appear as

$$
\dot{W}_{e}=-3 K \alpha \theta \operatorname{tr}(\dot{\varepsilon}),
$$

where $K$ is the bulk modulus and where $\operatorname{tr}(\cdot)$ denotes the trace of a tensorial quantity. The small strain tensor is denoted by $\varepsilon$, and assuming isotropy, the thermal strain tensor is written as $\alpha=\alpha 1$ with $\alpha$ being the coefficient of thermal expansion. The rate of temperature change is now found by combining (1) and (2) and subsequent integration gives the Kelvin formula for thermoelasticity under adiabatic conditions as

$$
\vartheta=-\frac{\alpha \theta_{0}}{\rho c} \operatorname{tr}(\sigma)
$$

where the notation $\vartheta=\theta-\theta_{0}$ was introduced to denote the relative temperature, $\theta_{0}$ being the reference temperature. In (3), it was also used that $\operatorname{tr}(\sigma)=3 K\left[\operatorname{tr}(\varepsilon)-3 \alpha\left(\theta-\theta_{0}\right)\right]$, valid since isotropic materials are considered. Note that under uniaxial conditions we have the identity $\operatorname{tr}(\sigma)=\sigma$.



Figure 1: Tensile specimen used in the experiments. The total length of the specimen is $150 \mathrm{~mm}$, the gauge length is $70 \mathrm{~mm}$ and the gauge diameter is $7 \mathrm{~mm}$. The bars were machined with $20 \mathrm{~mm}$ M10-threading at each end to allow fastening in the load-frame grips.

\section{Experimental Results}

A 100Cr6 steel was studied in two differently heat-treated conditions: as-received spheroidize-annealed material (SA) having a microstructure of ferrite, containing spheroidized carbides and also a material that was quenched and tempered (QT) at $610^{\circ} \mathrm{C}$ for 120 minutes resulting in a martensitic microstructure with a hardness of $170 \mathrm{HV}$, cf. [12]. Tensile specimens, cf. Figure 1, were prepared and uniaxially loaded in a servo-hydraulic load-frame at strain rates of $0.14 / \mathrm{s}$ and $0.0014 / \mathrm{s}$. The tensile bars had a total length of $150 \mathrm{~mm}$, gauge length $70 \mathrm{~mm}$, and gauge diameter $7 \mathrm{~mm}$. The bars were machined with $20 \mathrm{~mm}$ M10-threading at each end to allow fastening in the load-frame grips. K-type thermocouples were spot-welded to the specimen surfaces to monitor the change in temperature. Figures 2 and 3 show the stress-strain response and the corresponding changes in temperature for the SA and QT material, respectively. The graphs are averages of ten measurements. The $0.2 \%$ strain measure is indicated in the figures by dotted lines.The SA material exhibits a discontinuous yielding characterized by an upper initial yield point from which the subsequent yield stress drops as dislocations are freed from obstacles allowing them to move more easily. During a yield point, straining the dislocation density rapidly increases and work hardening gradually raises the stress level. This plateau is absent in case of the QT material which moves from the elastic to the plastic regime through essentially continuous yielding. The discontinuous yielding of the SA material makes it difficult to determine a distinct strain at which yielding occurs. However, considering the maximum temperature drop in Figure 2(b), a more clearly defined strain level is obtainable. In case of, the QT material continuous yielding occurs, making it seemingly easier to obtain the strain at which the elastoplastic limit is reached. Following [19], the mass density of the material is taken as $\rho=7850 \mathrm{~kg} / \mathrm{m}^{3}$, the specific heat as $c=584.13 \mathrm{~J} / \mathrm{kg} \mathrm{K}$, and the coefficient of thermal expansion as $\alpha=14.4 \times 10^{-6} 1 / \mathrm{K}$. This allows theoretical predictions of the temperature change using (3). Examining Figures 2 and 3 , it is possible to make an assessment of how well the $0.2 \%$ strain measure corresponds to the point where the thermoelastic temperature decrease reaches its maximum.

The experimentally obtained temperature drops are compared to those obtained by (3) in Table 1 From this table, it is seen that the calculated and measured temperature changes correspond relatively well except for the QT material at the higher strain rate. The difference in this case is approximately 50\%.

The strain and stress at the point of minimum temperature is compared to the standard $\sigma_{0.2}$-measure in Table 2 . It can be concluded that the yield stresses given by the $0.2 \%$ 


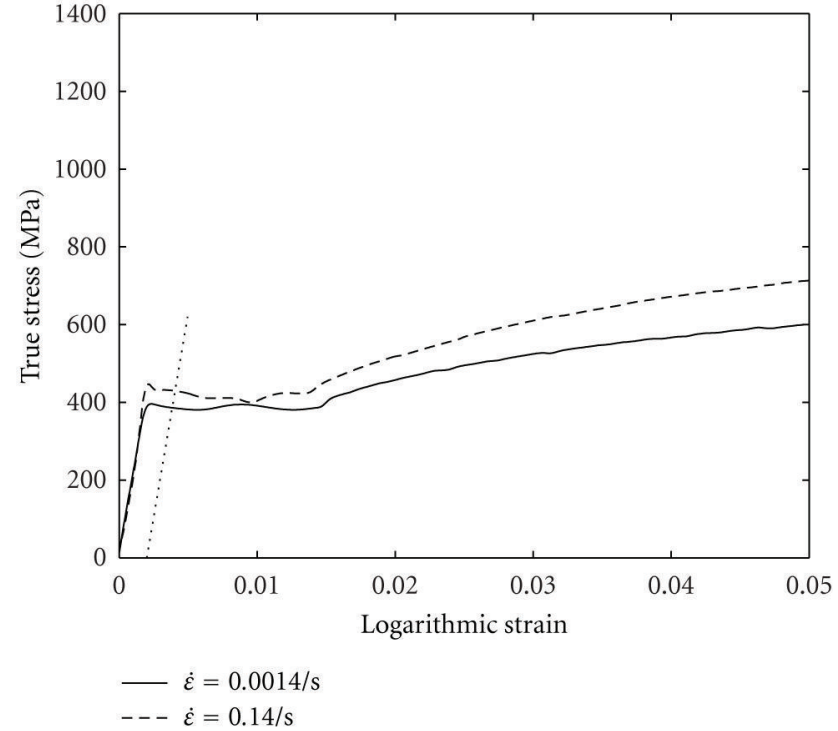

(a)

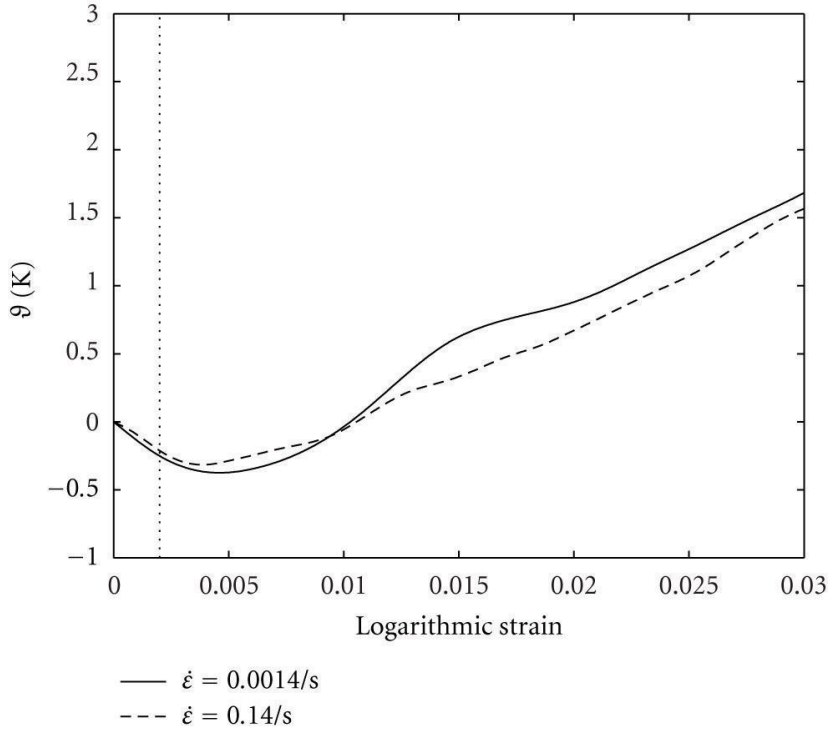

(b)

FIgURE 2: (a) Mechanical response and (b) temperature evolution in the SA material. Note that the $0.2 \%$ strain measure is indicated in the figures by a dotted line.

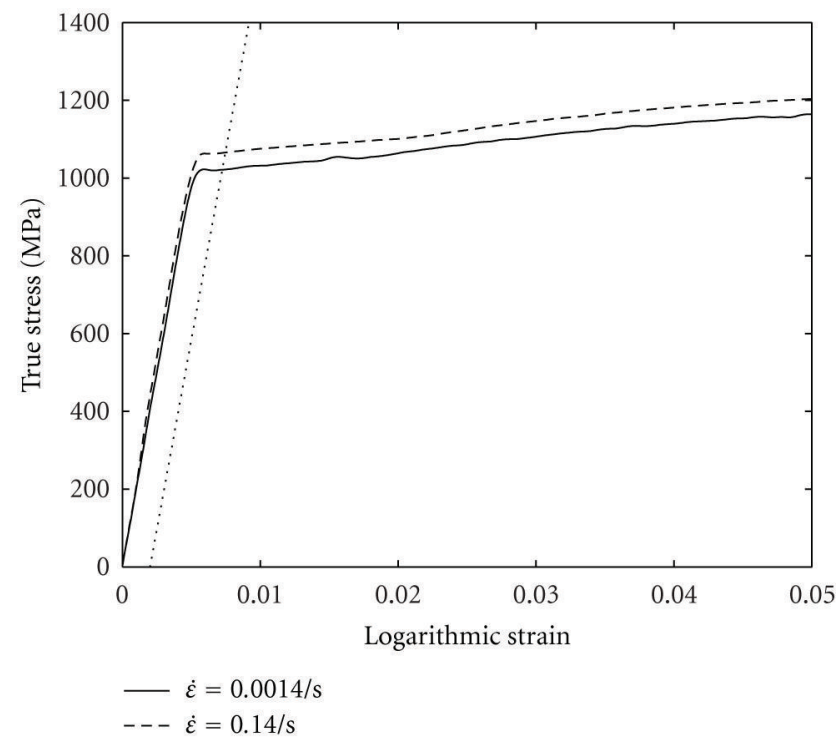

(a)

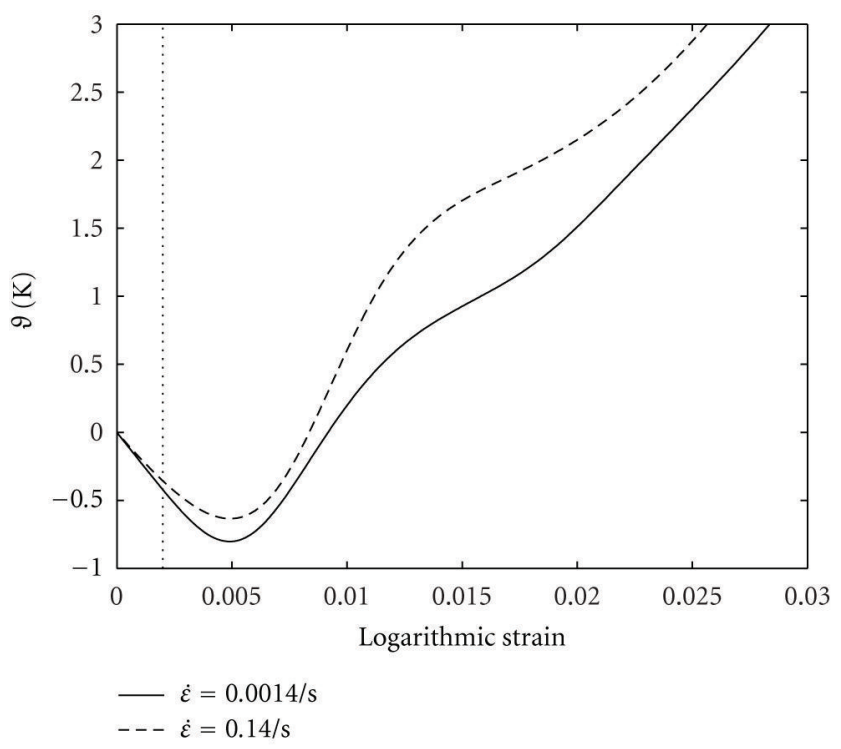

(b)

FIGURE 3: (a) Mechanical response and (b) temperature evolution in the QT material. Note that the $0.2 \%$ strain measure is indicated in the figures by a dotted line.

strain measure closely resemble the values obtained for the minimum temperature when considering the SA material. This is of course due to the yield plateau with a sharply defined stress level over an interval of corresponding yield strain. On the other hand, when considering the QT material, the $0.2 \%$ strain measure severely overestimates the yield stress as compared to that at the minimum temperature.
Yield stress differences in the order of $50 \mathrm{MPa}$ are found which undoubtedly are of interest in practical applications.

\section{Concluding Remarks}

The temperature evolution during uniaxial loading of two differently heat-treated conditions of $100 \mathrm{Cr} 6$ steel is studied. 
TABLE 1: Comparison of experimental and calculated estimates of the temperature drop at yielding for the two materials at two different strain rates. The experimental results are denoted by $\vartheta_{\text {exp. }}$. and the corresponding values obtained from (3) by $\vartheta_{\text {calc. }}$.

\begin{tabular}{lccc}
\hline Material & $\dot{\varepsilon}[1 / \mathrm{s}]$ & $\vartheta_{\text {exp. }}[\mathrm{K}]$ & $\vartheta_{\text {calc. }}[\mathrm{K}]$ \\
\hline SA & 0.0014 & -0.38 & -0.35 \\
SA & 0.14 & -0.32 & -0.40 \\
QT & 0.0014 & -0.80 & -0.98 \\
QT & 0.14 & -0.63 & -0.94 \\
\hline
\end{tabular}

TABLE 2: Comparison of yield point strain and stress for the two material conditions at two different strain rates. The strain and stress at yielding, corresponding to the maximum temperature drop, is denoted by $\varepsilon_{\text {yield }}$ and $\sigma_{\text {yield }}$, respectively. The yield stress at $0.2 \%$ inelastic strain is denoted by $\sigma_{0.2}$.

\begin{tabular}{lcccc}
\hline Material & $\dot{\varepsilon}[1 / \mathrm{s}]$ & $\varepsilon_{\text {yield }}$ & $\sigma_{\text {yield }}[\mathrm{MPa}]$ & $\sigma_{0.2}[\mathrm{MPa}]$ \\
\hline SA & 0.0014 & 0.0046 & 383 & 386 \\
SA & 0.14 & 0.0039 & 430 & 431 \\
QT & 0.0014 & 0.0049 & 963 & 1021 \\
QT & 0.14 & 0.0049 & 1016 & 1066 \\
\hline
\end{tabular}

By monitoring the minute temperature drop during elastic loading, a determination of the elastoplastic limit that is believed to be more adequate than the standard $0.2 \%$ strain measure is obtained. This is especially useful in the case of for example, annealed 100Cr6 where discontinuous yieldingand uncertain yield-point straining-occurs. Correspondingly, a quenched and tempered condition of $100 \mathrm{Cr} 6$ with continuous yielding reveals differences of up to $50 \mathrm{MPa}$ in yield stress when comparing the stress obtained by the $0.2 \%$ strain measure with the stress level at minimum temperature. The experimental results are compared to the theoretical temperature changes as predicted by the Kelvin formula with reasonable agreement in some cases but with up to a $50 \%$ difference in some, indicating shortcomings of the Kelvin formula.

\section{References}

[1] C. E. Bottani and G. Caglioti, "Thermal emission: a probe to identify the critical point of the elastoplastic transition," Materials Letters, vol. 1, no. 3-4, pp. 119-121, 1982.

[2] H. T. Lee and J. C. Chen, "Temperature effect induced by uniaxial tensile loading," Journal of Materials Science, vol. 26, no. 21, pp. 5685-5692, 1991.

[3] E. A. Pieczyska, S. P. Gadaj, and W. K. Nowacki, "Temperature changes in polyamide subjected to low cyclic deformation," Infrared Physics and Technology, vol. 43, no. 3-5, pp. 183-186, 2002.

[4] E. Grüneisen, "The thermic elongation and the specific heat of metals," Annalen der Physik, vol. 26, no. 6, pp. 211-216, 1908.

[5] E. Grüneisen, "The connection between compressibility, thermal expansion, atomic volumes and the atomu arms of metals," Annalen der Physik, vol. 26, no. 7, pp. 393-402, 1908.

[6] J. M. Dulieu-Smith and P. Stanley, "On the interpretation and significance of the Grüneisen parameter in thermoelastic stress analysis," Journal of Materials Processing Technology, vol. 78, no. 1-3, pp. 75-83, 1998.
[7] M. G. Beghi, C. E. Bottani, G. Caglioti, and A. Lenti, "Energy balance via thermal emission in copper under stress," Materials Letters, vol. 6, no. 4, pp. 133-137, 1988.

[8] A. Chrysochoos and G. Martin, "Tensile test microcalorimetry for thermomechanical behaviour law analysis," Materials Science and Engineering A, vol. 108, pp. 25-32, 1989.

[9] A. Chrysochoos, O. Maisonneuve, G. Martin, H. Caumon, and J. C. Chezeaux, "Plastic and dissipated work and stored energy," Nuclear Engineering and Design, vol. 114, no. 3, pp. 323333, 1989.

[10] S. P. Gadaj, W. K. Nowacki, and E. A. Pieczyska, "Changes of temperature during the simple shear test of stainless steel," Archive of Mechanics, vol. 4, no. 48, pp. 779-788, 1996.

[11] W. Oliferuk, W. A. Światnicki, and M. W. Grabski, "Rate of energy storage and microstructure evolution during the tensile deformation of austenitic steel," Materials Science and Engineering $A$, vol. 161, no. 1, pp. 55-63, 1993.

[12] H. Hallberg, K. Ryttberg, and M. Ristinmaa, "Model describing material-dependent deformation behavior in high-velocity metal forming processes," Journal of Engineering Mechanics, vol. 135, no. 4, pp. 345-357, 2009.

[13] W. Oliferuk, A. Korbel, and W. Bochniak, "Energy balance and macroscopic strain localization during plastic deformation of polycrystalline metals," Materials Science and Engineering A, vol. 319-321, pp. 250-253, 2001.

[14] G. C. Sih and D. Y. Tzou, "Heating preceded by cooling ahead of crack: macrodamage free zone," Theoretical and Applied Fracture Mechanics, vol. 6, no. 2, pp. 103-111, 1986.

[15] G. C. Sih and D. Y. Tzou, "Irreversibility and damage of SAFC-40R steel specimen in uniaxial tension," Theoretical and Applied Fracture Mechanics, vol. 7, no. 1, pp. 23-30, 1987.

[16] S. P. Gadaj, W. K. Nowacki, and E. A. Pieczyska, "Temperature evolution in deformed shape memory alloy," Infrared Physics and Technology, vol. 43, no. 3-5, pp. 151-155, 2002.

[17] E. Pieczyska, S. Gadaj, W. K. Nowacki, K. Hoshio, Y. Makino, and H. Tobushi, "Characteristics of energy storage and dissipation in TiNi shape memory alloy," Science and Technology of Advanced Materials, vol. 6, no. 8, pp. 889-894, 2005.

[18] L. Wu, S. Liu, Y. Wu, and H. Wu, "Changes in infrared radiation with rock deformation," International Journal of Rock Mechanics and Mining Sciences, vol. 39, no. 6, pp. 825-831, 2002.

[19] C. Mapelli, R. Venturini, and M. Boniardi, "Simulation and optimisation of an industrial process of sub-critical spheroidization annealing of a 100Cr6 steel," Scandinavian Journal of Metallurgy, vol. 34, no. 3, pp. 192-204, 2005. 

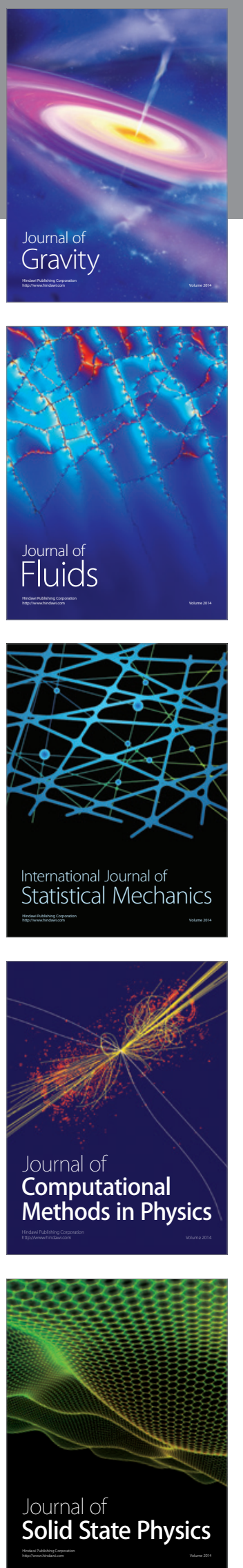


The Scientific World Journal



Submit your manuscripts at http://www.hindawi.com
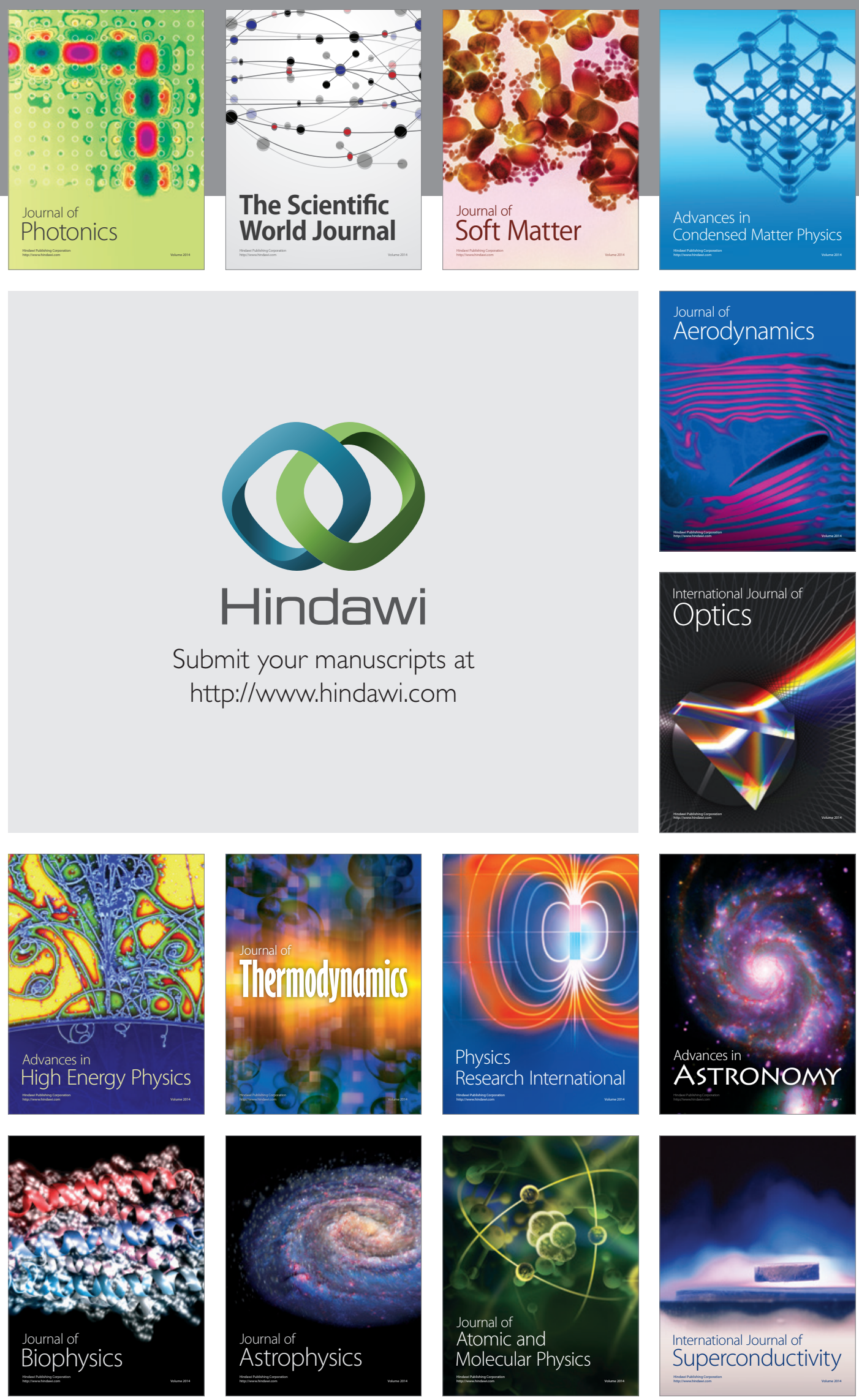
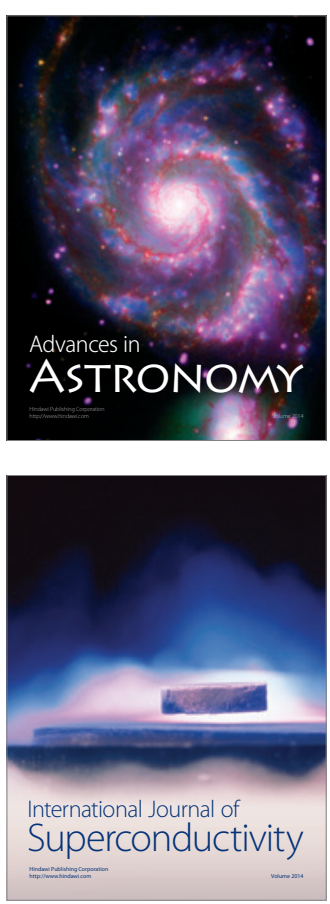